\title{
Research on the Jamming Effect of High Repetition Laser on Laser Guidance's Decoding
}

\author{
Shuai Zhang \\ Xi'an Research Institute of High-tech \\ Shaanxi, China \\ e-mail:zhangshuai_wonder@163.com \\ *Research supported by The Industrial Research Plan of \\ Shaanxi province under grant \#2012GY2-34. \\ Zhiguo Liu \\ Xi'an Research Institute of High-tech \\ Shaanxi, China \\ e-mail:lzgc@163.com
}

\author{
Shicheng Wang \\ Xi'an Research Institute of High-tech \\ Shaanxi, China \\ e-mail:wshcheng@vip163.com
}

\author{
Qian Zhao \\ Xi'an Research Institute of High-tech \\ Shaanxi, China \\ e-mail:zhaoqian_cool@163.com
}

\begin{abstract}
To estimate the effect of high repetition frequency laser jamming on laser seeker's distinguishing and locking stage for decoding process, two models are built to quantitatively study the jamming effect under the different coding systems, one is normalized cross-correlation function model, the other is jamming efficiency model. The simulation result indicates that, at the stage of code distinguishing, the jamming frequency is a dominant factor in affecting the jamming effect; The effect of high repetition frequency laser jamming seeker under the different coding system is different; The greater the seeker sampling interval is, the better the effect is. At the stage of code locking, the leading time of high repetition frequency laser to guidance signal is an important factor in affecting the jamming efficiency; The higher the jamming frequency and the wider the time gate's width is, the higher the average efficiency of interference is . High repetition frequency laser can completely jam the decoding process of the two stages when the laser frequency is high enough.
\end{abstract}

Keywords-High repetition laser; laser seeker; encoding and decoding; corss-correlation; jamming efficiency

\section{INTRODUCTION}

Recent years, laser semi-active guidance weapon plays a more and more important role in modern warfare, especially the excellent performance in the war of Afghanistan and Iraq which makes people gradually pay attention to laser semi-active guidance weapons and do research on them more deeply and precisely.

Laser jamming technology and anti-jamming technology of laser semi-active guidance weapons are like the spear and shield in the warfare, which always fight with each other and promote each other. The seeker decoding process can be divided into two stages: code distinguishing stage and code locking stage. In order to resistant to the interference and realize hitting at the targets accurately, at the stage of code distinguishing, most of the laser semi-active guidance weapons adopt laser coding techniques; At the stage of code locking, the laser semi- active guidance weapons usually put the time wave gate technology to use. In terms of jamming technology, the traditional passive laser jamming is greatly influenced by the weather and battlefield environment when in use. Laser responder interference needs to precisely identify the message of laser guided signal. Moreover, laser repeater jamming has delays and poor real-time performance, which can't adapt to the modern battlefield environment very well.

Laser high repetition frequency jamming is a new type of active jamming method, which utilizes high frequency laser to force into time wave gate and submerge guidance signals. Laser high repetition frequency jamming needn't identify laser encoding. In cooperation with the alarm system, it can actively jam laser guidance weapons, thus greatly improving the initiative and efficiency of the laser jamming.

However, seen from the present domestic study of high frequency interference, further research on the jamming mechanism and jamming effect of high repetition frequency laser is necessary. In this paper, on the assumption that high repetition frequency laser single pulse energy, pulse width and amplitude all meet the threshold, on basis of LFSR(linear feedback shift register) status code and US PIM(pulse interval modulation) code, at the stage of code distinguishing and locking, two models are built to quantitatively study the jamming effect under the different coding systems, one is normalized cross correlation function model, the other is jamming efficiency model. Then the main factors influencing the high repetition laser jamming effect can be obtained.

\section{ANALYSIS ON LASER CODING AT HOME AND ABROAD}

Domestic laser coding can mainly be divided into short cycle code and code for a long period of time. Short cycle code $^{[1]}$ mainly includes accurate frequency code, two variable space code, PCM(pulse code modulation) and finite-bit random cycle code. Short cycle code is easy to 
recognize and has poor anti-jamming performance because of its simple coding; Code for a long period of time mainly contains arithmetic type code, LFSR modulation code and LFSR status code. And laser coding abroad is mainly in the United States where it is the most typical.US PIM code is an earlier code, which is applied to the early American precision-guided weapons and has a very good representative in the laser guidance weapons. Because the regularity of arithmetic type code and LFSR modulation code is stronger and easier to identify and reproduce, while the pseudo randomness and anti-jamming performance of LFSR status code and US PIM code is better. Based on the latter two code, this paper carries out research on high repetition frequency laser jamming performance of the two kinds of code, and then evaluates high repetition frequency laser jamming effect at the two different decoding stage.

\section{A. LFSR Status Code}

LFSR status code bases on the state transition diagram $^{[2]}$ and makes use of linear state feedback shift register to produce $(0,1)$ sequence. Setting $\Delta T_{c}=41.28 \mathrm{~ms}$, clock cycle $\Delta T_{0}=0.1 \mathrm{~ms}$ and $a_{0}=01011010$ in LFSR status code, LFSR status code pulse interval sequence is shown in Fig .1:

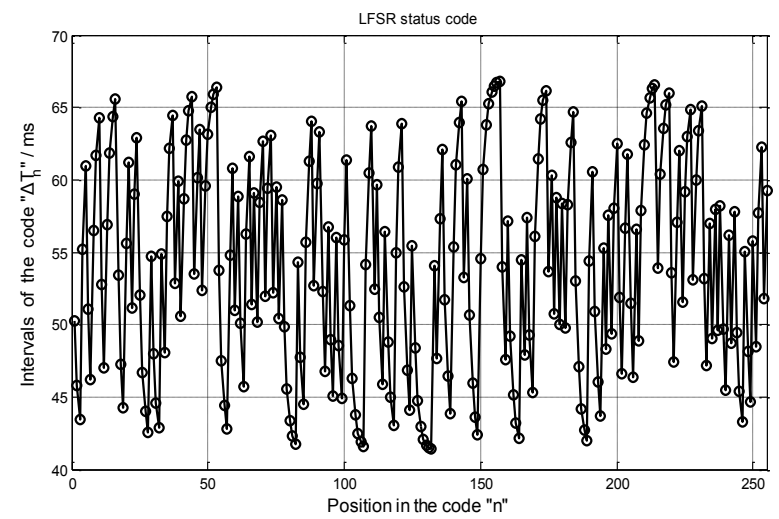

Figure 1. The pulsed interval sequence of LFSR status code

\section{B. US PIM code}

US PIM code is an advanced coding way applied to American laser guidance weapons, which is also a pseudo random $\operatorname{code}^{[3]}$ by improving the linear congruence algorithm to realize composite encryption technology. Its coding can be divided into three steps:

Fristly, asume that the pulse interval is $\Delta T=\left\{\Delta T_{m} \mid 1 \leq m \leq 256\right\}$.Encode the guidance signal pulse interval, namely make $\Delta T=\Delta T_{c}+N_{m} \cdot \Delta t_{0}$, then get the coding result $N_{m}$ (can be as a binary or decimal number).

Secondly, based on the linear congruence algorithm in cryptography to generate pseudo random address $A D D=\left\{A D D_{m} \mid m \geq 1\right\}$, the method is:

$$
\left\{\begin{array}{l}
A D D_{1}=A D D_{0} \\
A D D_{m}=\left(A D D_{m-1}+\Delta_{m-1}\right)(\bmod 256)
\end{array}\right.
$$

In the formula, $\Delta=\Delta_{1} \Delta_{2} \cdots \Delta_{m-1}$ is a given secret key sequence.
Thirdly, using the affine encryption algorithm realizes the address and pulse interval mapping one by one, namely $N_{m}=E\left(A D D_{m}\right)$.

In the formula, $E(\cdot)$ is the affine encryption algorithm, which can also use nonlinear algorithms such as $\mathrm{P}$ permutation and $\mathrm{S}^{[3]}$ box algorithms.

Take $\Delta T_{c}=41.28 \mathrm{~ms}, \Delta t_{0}=0.1 \mathrm{~ms}$, secret key $\Delta=\{1,2,3 \mathrm{~L} 255\}, N_{m}=A D D_{m}$, suppose $A D D_{0}=79$. US PIM code is shown in Fig .2:

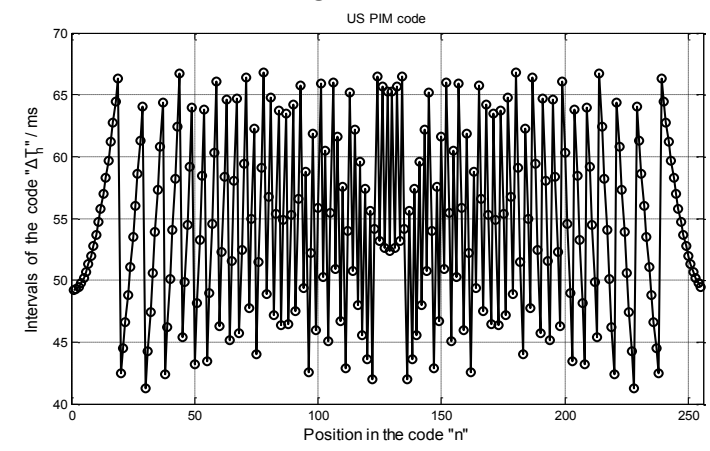

Figure 2. The pulsed interval sequence of US PIM

From the above picture which displays the two coded pulse (LFSR status code and US PIM code) intervals changing with the amount of pulses, it can come to a conclusion that both of the two codes are pseudo random codes for a long period of time, and US PIM code has the better pseudo randomness and stronger anti-interference ability. And these two kinds of codes are easily implemented on hardware, and easy to be applied to weapons and equipment.

\section{RESEARCH ON MODELING OF HIGH REPETITION FREQUENCY LASER JAMMING}

\section{A. Research on Modeling of Code Distinguishing Stage}

Take advantage of the nature that related pulse subsequence autocorrelation function is equal to 1 to realize the laser seeker decoding of code distinguishing stage. Laser guidance weapons generally use hardware method to implement the physical decoding ${ }^{[4]}$ on account of digital correlator equipped with the sliding matching algorithm.

Because the high repetition frequency jamming laser can be fired before the guidance signal emission in conjunction with the alarm system ${ }^{[5-7]}$, it can be made sure that high repetition frequency jamming signal advances guidance signal into the seeker information processing system.

From this, define the normalized cross- correlation function:

$$
r_{g z}=\frac{1}{M} \sum_{k=0}^{N-1} g(n) y(n+k)
$$

In the above equation, regard $g(n)$ as high frequency pulse sequence, $y(n)$ as the laser preset coded pulse subsequence, $N$ as the length of the two sequences, $M$ as the number of coded signal in the subsequence. 
Realize sliding matching process by means of crosscorrelation function. If $r_{g z}=1$, the match is successful, which means that high repetition frequency pulse signal can completely interference seeker and partial ${ }^{[8-10]}$ the laser guidance weapons; if $r_{g z}$ is closer to 1, the degree of match is higher, which means that high frequency pulse sequence contains the more jamming signal which has the same phase as the guidance pulse and interferes with the coding identification process greater, so as to decoy and block the seeker information processing unit ${ }^{[10]}$. Therefore the normalized cross-correlation function can reflect the interference performance ${ }^{[9]}$ of jamming laser to laser codec.

\section{B. Jamming Efficiency Modeling of Code Locking}

Once the guidance signal has been detected, the laser seeker matches the guidance signal and adopts the timewave gate technology to detect the subsequent pulses at the stage of code locking, so as to filter out the jamming signal effectively. When other influencing factors meet the requirements of the corresponding threshold, the jamming effect of high repetition frequency laser is related to the jamming pulse frequency and the width of time wave gate at the same time. So in this article, the number of pulses in phase represent the jamming ability and the jamming efficiency model is set up. The quantitative values of the jamming efficiency are utilized to represent the jamming effect. This model is as follows:

$$
\eta_{G}(m)=\frac{1}{N} \sum_{n=1}^{N} X(n) \cdot G(n+m)
$$

In the above equation, $X(n)$ and $G(n)$ respectively stand for the 0,1 sequence of guidance signal and high repetition jamming signal after discretization. $m$ stands for pulse amount of high repetition frequency pulse advancing guidance pulse $N$ is the guidance pulse number in sampling time. $\eta_{G}(m)$ is the jamming efficiency when ahead of $m$ pulses.

At the same time, the number of the high repetition frequency pulse sequence advancing the guidance pulse sequence is random, and assume that $m$ is evenly distributed of $[0, M]$. The average interference efficiency model is set up as follows:

$$
\bar{\eta}_{G}=\frac{\sum_{m=0}^{M} \eta_{G}(m)}{M+1}
$$

From the perspective of laser codec, the average efficiency of anti-jamming is:

$$
\bar{\eta}_{G K}=1-\frac{\sum_{m=0}^{M} \eta_{G}(m)}{M+1}
$$

The larger $\bar{\eta}_{G K}$ is, the stronger anti-interference ability of high repetition frequency pulse under a certain frequency is.

\section{JAMMING EFFECT SIMULATION EXPERIMENT}

On the basis of the above analysis and model, the jamming effect of high repetition frequency pulse interfering with LFSR status code and US PIM code is simulated at the stage of code distinguishing. Because the code pattern has been identified at the code locking stage and jamming effect has nothing to do with the specific code pattern, example of LFSR status code and experiment on the jamming effect is taken.

\section{A. Code Distinguishing Stage}

Based on the seeker sliding matching algorithms at the stage of code distinguishing, the result of cross-correlation function presents the matching degree between high repetition pulse sequence and the preset pulse sequence.

(1) Adopt LFSR status code when simulation, and take $\mathrm{M}=10$, sampling interval $\Delta t=0.01 \mathrm{~ms}$, the normalized cross-correlation results are shown in the Fig .3:

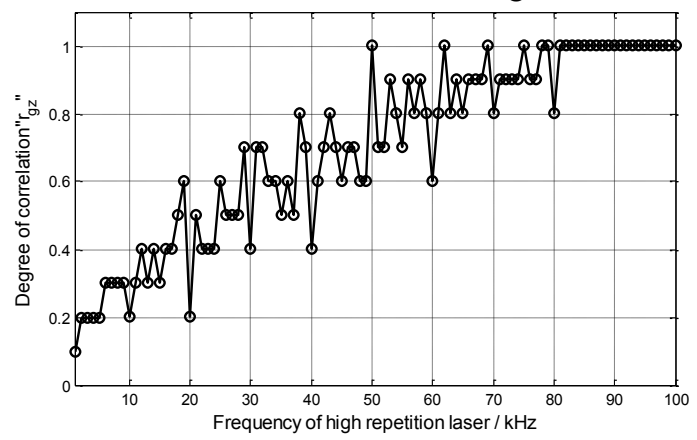

Figure 3. The result of corss-correlation of high repetition frequency laser jamming on LFSR status code

(2) Adopt US PIM code when simulation, and take $\mathrm{M}=10, A D S_{0}=79$, sampling interval $\Delta t=0.01 \mathrm{~ms}$, the normalized cross-correlation results are shown in the Fig .4:

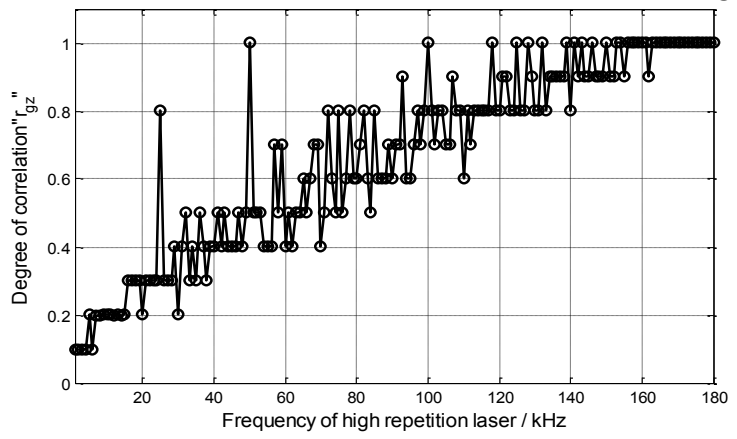

Figure 4. The result of corss-correlation of high repetition frequency laser jamming on US PIM( $\Delta t=0.01 m s)$

(3) Adopt US PIM code when simulation, take $\mathrm{M}=10, A D S_{0}=79$, sampling interval $\Delta t=0.02 \mathrm{~ms}$, the normalized cross-correlation results are shown in the Fig .5:

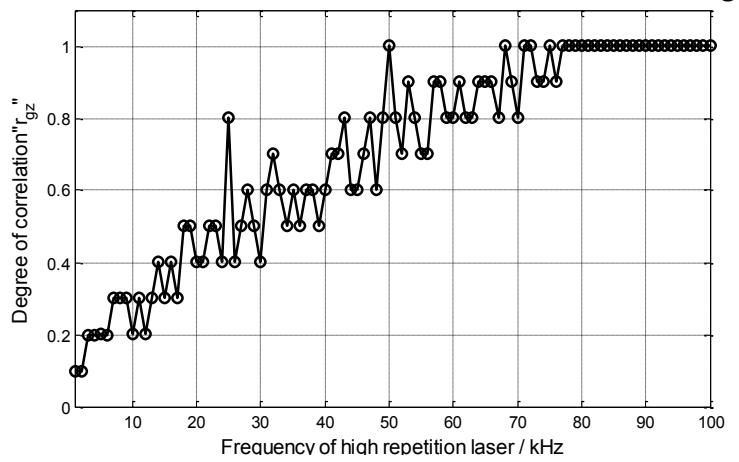

Figure 5. The result of corss-correlation of high repetition frequency laser jamming on US PIM( $\Delta t=0.02 m s)$ 
The simulation results show that: high repetition frequency pulse can completely jam guidance pulse when it reaches a certain value. Then the jamming pulse interval is very short, and there is at least one jamming pulse at each sampling interval; Comparing Fig .3 with Fig .4, it shows that high repetition frequency pulse can completely jam LFSR status code when it reaches $81 \mathrm{kHz}$, while only when high repetition frequency pulse reaches $164 \mathrm{kHz}$ can it interfere with US PIM code completely, so we can come to a conclusion that the effect of high repetition frequency laser pulse jamming LFSR status code is better than US PIM code, which is in accordance with the analysis results on pseudo-randomness of the two codes; Comparing Fig .4 with Fig .5,it can be observed that during the discretization the smaller the sampling interval is, the stronger antiinterference ability is. So improving the distinguishing and recognition performance of the circuit inside the seeker can improve the anti-jamming ability. In terms of the sampling interval in Fig .3 and Fig .4, high repetition frequency of $100 \mathrm{kHz}$ can achieve full interference with two kinds of code when sampling interval is equal to $0.01 \mathrm{~ms}$, then high repetition frequency of $50 \mathrm{kHz}$ can achieve full interference with two kinds of coding when sampling interval is equal to $0.02 \mathrm{~ms}$ in Fig .5. This suggests that when the sampling interval is equal to the reciprocal of high repetition frequency, the jamming effect has nothing to do with the code pattern.

\section{B. Code Locking Stage}

Jamming efficiency is not only related to high repetition pulse frequency, but also related to the width of time wave gate at the stage of code locking. Based on LFSR status code in part II, two groups of simulation experiment in a cycle which consists of the ten pulses are carried out. According to the groups of the simulation results, the average jamming efficiency can be obtained at different width of time wave gate and different high repetition frequency. Laser guidance weapons basically adopt real-time wave gate, the width of which is generally $10 \mu s \sim 50 \mu s$, so real-time wave width of $10 \mu s$ and $20 \mu s$ are simulated respectively.

(1)Take width of time wave gate $\Delta t_{g}=10 \mu \mathrm{s}$, high repetition frequency $f=1 k, f=8 k, f=20 k$, $f=40 k$, then the jamming efficiency can be obtained at different time that high repetition frequency pulse advances guidance pulse. As shown in the Fig .6:
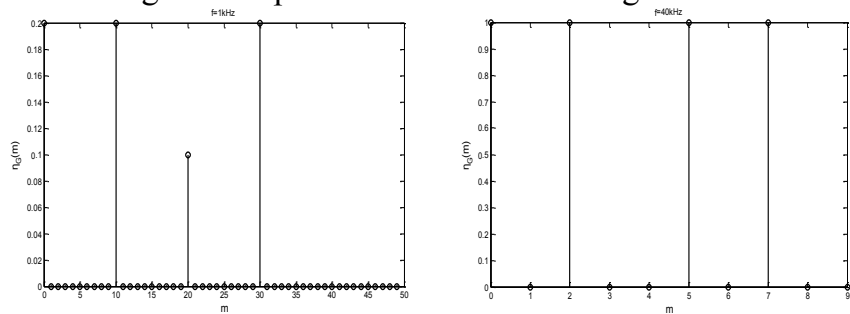
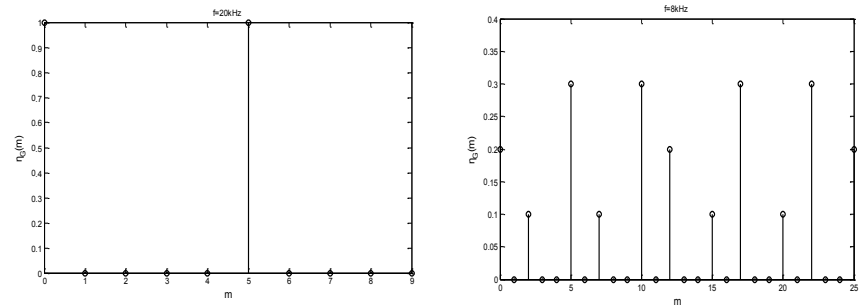

Figure 6. Wave gate is $10 \mu \mathrm{s}$,jamming frequency result in jamming efficiency at different leading time

(2)Take width of time wave gate $\Delta t_{g}=25 \mu \mathrm{s}$, high repetition frequency $f=1 k, f=8 k, f=25 k$, $f=40 k$, then the jamming efficiency can be obtained at different time that high repetition frequency pulse advances guidance pulse. As shown in the Fig .7:
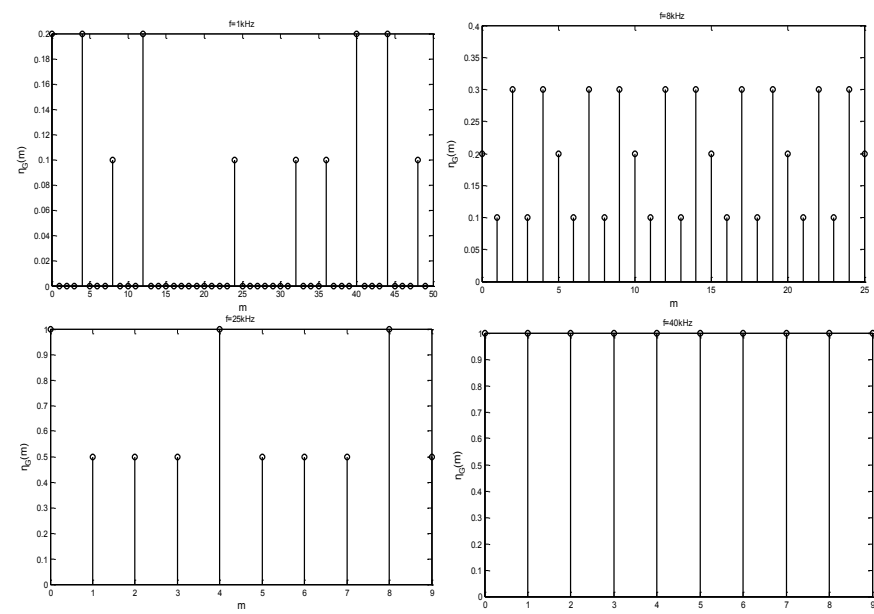

Figure 7. Wave gate is $25 \mu s$, jamming frequency resule in jamming efficiency at different leading time

From Fig .6 and Fig .7, the following conclusions can be drawn: at the stage of code locking, when the width of time wave gate and high repetition frequency are fixed, different leading time corresponds to different jamming effect, and there exists appropriate leading time making jamming effect best. In Fig .6 when $f=20 k$ and $m=5$, and in Fig .7 when $f=25 k$ and $m=4$, the jamming can achieve the best effect.

(3)According to the results above, using the average jamming efficiency model calculates the average jamming efficiency at different high repetition frequency pulse when the width of time wave gate is equal to $10 \mu \mathrm{s}$ and $25 \mu s$. 


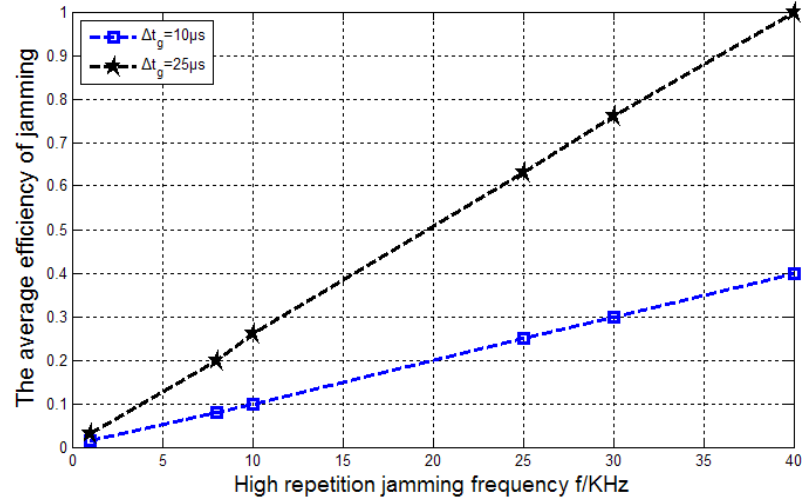

Figure 8. High repetition frequency result in jamming efficiency at different gate's width

By the above simulation curve, the average jamming effect is related to the width of time wave gate and jamming frequency. The wider the time wave gate and the higher the high repetition frequency, the better the average jamming effect is. The average jamming efficiency $\bar{\eta}_{G}$ of high repetition frequency pulse has a linear relationship with the width of time wave gate and high repetition frequency, satisfying the following equation:

$$
\bar{\eta}_{G}=\Delta t_{g} \cdot f_{G}
$$

As a result, at the stage of seeker code locking, after setting wave door width, when it is $\Delta t_{g}^{\prime}$, the anti-jamming efficiency is obtained.

$$
\bar{\eta}_{G K}=1-\Delta t_{g}^{\prime} \cdot f_{G}
$$

Therefore, at this stage, anti -jamming ability mainly depends on the width of time wave gate. Setting up a narrow wave gate is helpful to improve the jamming performance of laser seeker.

\section{JAMMING EFFECT ANALYSIS}

The main task of seeker at code distinguishing stage is to recognize guidance laser. During the process of recognition, seeker information processing component distinguishes guidance pulse through the signal correlation algorithm. The essence of the matching algorithm is to complete the similar judgment between the signals received by seeker and preset signals after discretization, and if the result is greater than a certain threshold, it is considered to be guidance signal. The simulation results show that at the stage of coding distinguishing, if high repetition frequency reaches a certain value, it can fully obtain the same signal with phase of guidance, which is the advantages of high repetition frequency jamming; Also, under the different ways of encoding, the same high repetition frequency contains different signal with phase, this is why the anti-jamming performance of US PIM code is better than LFSR status code in the simulation results.

During the stage of code locking, the premise of high repetition frequency pulse playing effect is that jamming signals can be forced into time-wave gate and jam guidance pulse according to the corresponding pulse sampling technology. In this paper, the essence of the jamming efficiency model is to simulate the jamming probability of high repetition frequency pulse forcing into time wave gate when the width and appropriate leading time is set. The simulation results reveal that average jamming efficiency has a linear relationship with the width of time wave gate and high repetition frequency, which is the same as the actual working status of laser seeker.

\section{CONCLUSION}

Through the analysis of laser coding techniques and seeker anti-jamming technology, LFSR status code and US PIM code are applied to implement simulation of high repetition frequency pulse jamming guidance pulse at code distinguishing stage. Jamming efficiency model is set up to study jamming effect of code locking stage. At the stage of code distinguishing, jamming effects are simulated under different encoding. The results show that jamming effects is connected with jamming frequency, sampling intervals and coding pattern, and jamming effects have something to do with jamming frequency and the width of time wave gate at code locking stage.

In addition, jamming effect should also have relevance to pulse energy, pulse width, amplitude, the target reflectivity and other factors. These need to be taken into consideration in a follow-up study. Jamming effect is essentially determined by the seeker internal hardware circuit, so it is critical for seeker information processing components to improve the ability of handling signals. Simulation results of this paper can provide reference for the improvement of the seeker hardware circuit.

\section{REFERENCES}

[1] W. P. Wang, and J Liu, Introduction to unmanned air vehicle route planning methods[J], FLIGHT DYNAMICS, 2010, 28(2):6-10.

[2] ZHONG Zhong-liang, HE Yong-qiang, ZHOU Bing, YING Jia-ju. Study on identification technique of guiding laser code information [J]. LASER\&INFRARED, 2011, 41(6):660-663. (in Chinese)

[3] Wei Wen-jian. Key Techniques and System Study on HWIL Simulation for Laser Guidance and Electro-optical Countermeasures[D]. Changsha: PHD thesis, National University of Defese Technology, 2010. (in Chinese)

[4] Wade Trappe TawrenceC.Washington. Introduction to Cryptography with Coding Theory Second edition[M]. China: POSTS\& TELHCOM PRESS, 2008.

[5] Zhao Qian,Liu Zhi-guo, Wang Shi-cheng,et al.Reserch on the effect of high-repetition-frequency laser jamming on different coding systems[J]. LASER \& INFRARED, 2014,44(4): 414 418. (in Chinese)

[6] Wei Wen-jian, Qin Shi-qiao, Zhan De-jun, et al. Research on laser encoding laser semi-active homing guidance[J]. LASER \& INFRARED, 2008, 38(12): 1199 1203. (in Chinese)

[7] Wang Wei-feng, Liu Zhi-guo, Wang Shi-cheng, et al. Design of energy simulation system for laser guided weapons[J]. LASER TECHNOLOGY, 2012, 36 (6): 844 848 . (in Chinese)

[8] Xue Jian-guo, Chen Yong. Research on the jamming effect of the high repetition laser to the laser guidance[J]. AERO WEAPONRY, 2006,(3):30 32. (in Chinese)

[9] Li Hui, Li Yan, Liu Bing-feng, et al. Status, development and key technique analysis of laser jamming technology [J]. CHINESE JOURNAL OF LASERS,48,081407(2011). (in Chinese)

[10] Zhu Chen-cheng, Nie Jin-song, Tong Zhong-chen. Analysis on the mode of high repetition laser jamming $[\mathrm{J}]$. Infrared and Laser Engineering, 2009,38(6):1060 1063. (in Chinese) 